\title{
ZERO-ONE LAWS FOR SPARSE RANDOM GRAPHS
}

\author{
SAHARON SHELAH AND JOEL SPENCER
}

\section{INTRODUCTION AND SUMMARY}

Let $G=G(n, p)$ denote the random graph with $n$ vertices and edge probability $p$. Let GRA denote the first order theory of graphs, as described at the end of this section. For any $p \in[0,1]$ and any statement $A$ in GRA set

$$
f(n, p, A)=\operatorname{Pr}[G(n, p) \text { has } A] .
$$

Fagin [6] and, independently, Glebskii et al. [7] proved that for all $p, A$,

$$
\lim _{n \rightarrow \infty} f(n, p, A)=0 \text { or } 1 .
$$

For random graph theorists (see, e.g., Bollobás [1] for general reference) $p$ "any constant" is not the only, not even the most interesting case. Rather, they consider $p=p(n)$, a function approaching zero. In their seminal paper, Erdós and Rényi [5] showed that for many interesting $A$ there is a function $p(n)$, which they called a threshold function, so that if $r(n) \ll p(n)$ then $f(n, r(n), A) \rightarrow 0$ while if $p(n) \ll r(n)$ then $f(n, r(n), A) \rightarrow 1$. (Notation: $p \ll r$ means $\lim p / r=0$. All limits are as $n$ approaches infinity.) Let us say $p=p(n)$ satisfies the Zero-One Law if for all $A$ in GRA, $\lim f(n, p, A)=0$ or 1 . We shall partially characterize those $p=p(n)$ which satisfy the Zero-One Law.

When $p<n^{-1+o(1)}$, the "very sparse" range, we give in $\S 2$ a nearly complete answer.

Theorem 1. If either

(i) $p \ll n^{-2}$,

(ii) for some integer $k, n^{-1-1 / k} \ll p \ll n^{-1-1 /(k-1)}$,

(iii) $n^{-1-\varepsilon} \ll p \ll n^{-1}$ for all $\varepsilon>0$,

Received by the editors January $15,1987$.

1980 Mathematics Subject Classification (1985 Revision). Primary 05C80, 03C10.

Research of first author partially supported by U. S.-Israel Binational Science Foundation.

Research of second author partially supported by National Science Foundation (U.S.). 
(iv) $n^{-1} \ll p \ll n^{-1} \log n$, or

(v) $n^{-1} \log n \ll p \ll n^{-1+\varepsilon}$ for all $\varepsilon>0$,

then $p$ satisfies the Zero-One Law.

The functions $n^{-2}, n^{-1-1 / k}, n^{-1}$, and $n^{-1} \log n$ are all known threshold functions. In this range the Zero-One Law holds as long as $p$ falls "between the cracks" of the spectrum of threshold functions. For completeness we mention that Fagin's proof actually gives that if $p \gg n^{-\varepsilon}$ for all $\varepsilon>0$ then $p$ satisfies the Zero-One Law.

It had been conjectured by the second author that if $p(n)$ were reasonably smooth then $\lim f(n, p, A)$ would exist. In $\S 3$ we give the following counterexample to that conjecture.

Theorem 2. There is a statement $A$ so that for any $p=n^{-1 / 7} q^{1 / 7}$ with $n^{-1 / \log _{5} n}<q<\log n / \log _{5} n$ the function $f(n, p, A)$ does not approach a limit in $n$. Moreover, if we restrict $n$ to satisfy $\log ^{*} n \equiv 25 \bmod 100$ then $f(n, p, A) \rightarrow 1$ whereas if we restrict $n$ to satisfy $\log ^{*} n \equiv 75 \bmod 100$ then $f(n, p, A) \rightarrow 0$.

Here $\log _{5} n=\log \log \log \log \log n$ and $\log ^{*} n$ is the least $k$ so that the sequence $u_{0}=n, u_{i+1}=\log u_{i}$ has $u_{k}<2$ where, for convenience, we assume all logs are to base two.

The statement $A$ will be (theoretically) explicitly given; it allows us to essentially interpret a segment of arithmetic on an appropriate intersection of neighborhoods. The exponent $-1 / 7$ and the expression $\log _{5} n$ are conveniences; for any rational $r, 0<r<1$, we may find an $A$ so that for $p$ appropriately near $n^{-r} f(n, p, A)$ does not approach a limit in $n$.

In $\S 4$ we prove

Theorem 6. If $\alpha$ is irrational then $p=n^{-\alpha}$ satisfies the Zero-One Law.

In the final section we take a dynamic view and examine the behavior of $\operatorname{Pr}[A]$ as $p$ evolves from 0 to 1 through the functions of $n$. We define a spectrum $\operatorname{Spec}(A)$, giving those spots where $\operatorname{Pr}[A]$ changes, and give a partial characterization of the possible spectra of first order statements.

The first order theory of graphs, GRA, consists of two binary predicates, equality and adjacency, with adjacency assumed symmetric and antireflexive. The formulae of GRA are built up from atomic formulae of type either $x_{i}=x_{j}$ or $x_{i} \sim x_{j}$, where $\sim$ denotes adjacency, using the logical connectives $\wedge$ (and), $\vee$ (or), $\neg$ (not), $\rightarrow$ (implies) and also the quantifiers $\exists$ (there is), $\forall$ (for all). Here the $x_{i}$ are interpreted as the vertices of the underlying graph ("the universe") so quantifiers may be read "there is a vertex $x_{i}$ such that" and "for every vertex $x_{i}$ there holds." Formulae in which all occurring variables are bounded by quantifiers are called closed formulae or sentences. One method, the method we employ, to prove results on formulae is by induction along the 
way it is built up. By propositional calculus every formula is equivalent to another built just by using $\neg, \vee$, and $\exists$.

GRA is our framework throughout this paper. In GRA we can make statements such as " $G$ contains a triangle" or " $G$ has no isolated points." Other statements, such as " $G$ is planar" or " $G$ is Hamiltonian," cannot be made in this language. This restriction may appear artificial to many graph theorists who feel that appropriate Zero-One Laws hold for a much wider class of "natural" graph-theoretic statements. There are logical pitfalls. Kaufmann and Shelah [9] have shown that even Fagin's Theorem does not hold in monadic second order logic. It would be interesting to find a logical structure stronger than GRA in which, for example, the statements above could be made and for which analogs to Fagin's Theorem and our results could be proven.

\section{VERY SPARSE GRAPHS}

Here we prove Theorem 1, which is really five theorems. The plan, due to Fagin, is identical in all cases. If we can find a family $\mathscr{A}$ of statements in GRA such that

(i) $\lim f(n, p, A)=1$ for all $A \in \mathscr{A}$,

(ii) $\mathscr{A}$ is complete, i.e., every statement of GRA is either provable or refutable from $\mathscr{A}$,

then $p$ satisfies the Zero-One Law. For let $B$ be any statements in GRA. Suppose $B$ is provable from $\mathscr{A}$. By compactness (the finiteness of proof) some finite number $A_{1}, \ldots, A_{m}$ of statements in $\mathscr{A}$ will imply $B$. As

$$
f\left(n, p, A_{1} \wedge \cdots \wedge A_{m}\right) \geq 1-\sum_{i=1}^{m} f\left(n, p, \neg A_{i}\right),
$$

$\lim f\left(n, p, A_{1} \wedge \cdots \wedge A_{m}\right)=1$ and hence $\lim f(n, p, B)=1$. If $B$ is not provable then by completeness $\neg B$ is provable, $\lim f(n, p, \neg B)=1$ and $\lim f(n, p, B)=0$.

In each case we give a suitable $\mathscr{A}$. Verification of (i) is then an elementary exercise in random graphs. To show (ii) it suffices, by the Gödel Completeness Theorem, to show

(iii) all countable models of $\mathscr{A}$ are isomorphic.

Fagin let $\mathscr{A}$ be the set of extension axioms $A_{r, s}:$ For all distinct $x_{1}, \ldots, x_{r}$, $y_{1}, \ldots, y_{s}$ there is a point $z$ adjacent to all of the $x_{i}$ and none of the $y_{j}$. The countable $G$ satisfying all $A_{r, s}$ are isomorphic by a simple "back and forth" argument.

Case 1. $p \ll n^{-2} . \mathscr{A}$ consists of the single statement "There are no edges."

Case 2. $n^{-1-1 / k} \ll p \ll n^{-1-1 /(k-1)}$. For each tree $T$ on at most $k$ points (including the tree on one vertex) and each $r$ let $A_{T, r}$ be the statement " $G$ has (at least) $r$ components $T$." Let $B$ be the statement " $G$ does not contain $k+1$ points whose internal edges contain a tree." Let $C$ be the statement " $G$ 
has no cycles of size at most $k$." Let $\mathscr{A}$ be all of the above statements. A countable model $G$ must then consist precisely of a countable number of copies of every tree $T$ of size at most $k$ and (iii) is satisfied.

In the remaining cases we cannot show (iii) but rather

(iv) all countable models of $\mathscr{A}$ are elementarily equivalent.

Proof of (iv) in the cases below can be given in various ways, see, e.g., Marcus [8], particularly Lemma 2.1 .

Case 3. $n^{-1-\varepsilon} \ll p \ll n^{-1}$ for all $\varepsilon>0$. For each tree $T$ and $r$ let $A_{T, r}$ be the statement "There are (at least) $r$ components $T$." For each $r$ let $C_{r}$ be the statement "There are no cycles of length $r$."A countable model $G$ then consists of countably many copies of each finite tree plus possibly some infinite trees.

Case 4. $n^{-1} \ll p \ll n^{-1} \log n$. For each finite tree $T$ let $A_{T, r}$ be the statement "There are (at least) $r$ components $T$." For all $m \geq 3, r$ let $B_{m, r}$ be the statement "There are (at least) $r$ cycles of size $m$." For all $m$ let $C_{m}$ be the statement "There do not exist $m$ vertices containing (at least) $m+1$ internal edges." For all $m, r$ let $D_{m, r}$ be the statement "There do not exist $m$ vertices with exactly $m$ internal edges with one vertex having (total) degree precisely $r$." A countable model $G$ consists of countably many copies of each finite tree, for each $m$ countably many components consisting of a unique cycle of length $m$ with all vertices having infinite degree plus possibly some infinite trees.

Remark. N. Pippenger (San Jose) has noted that the case $p=\Theta\left(n^{-1} \ln n\right)$ contains a doubly infinite sequence of "tight" threshold functions. Let $\mathscr{F}$ be the class of functions

$$
f(n)=\frac{1}{K} \frac{\ln n}{n}+\frac{L}{K} \frac{\ln \ln n}{n}
$$

where either $K=1, L=0$ or $K \geq 2, L \geq K-1$, both integral. Say $p(n)<^{*} f(n)$ if $\lim n(f(n)-p(n))=+\infty$. The Zero-One Law holds in this range for $p(n)$ if for every $f(n) \in \mathscr{F}$ either $p(n)<^{*} f(n)$ or $f(n)<^{*} p(n)$.

Case 5. $n^{-1} \log n \ll p \ll n^{-1+\varepsilon}$ for all $\varepsilon>0$. For each $r$ let $A_{r}$ be the statement "There are no points of degree $r$." Let $B_{r}$ be the statement "No $r$ points have $r+1$ internal edges." For each $m \geq 3, r$ let $C_{r, m}$ be the statement "There exist $r$ cycles of length $m$." Let $G_{m}$ be that unique connected graph containing a single cycle of length $m$ with all points of infinite degree. Let $G_{0}$ be the tree with all points of infinite degree. A countable model $G$ consists of countably many copies of each $G_{m}$ and possibly some copies of $G_{0}$.

\section{INTERPRETING ARITHMETIC}

The proof of Theorem 2 proceeds in three stages. First we define $A$. Second we show that the random graph almost always has certain properties. Finally, 
we show that when $G$ has these properties $A$ holds when $\log ^{*} n \equiv 25 \bmod 100$ and $A$ does not hold when $\log ^{*} n \equiv 75 \bmod 100$.

Statement $A$ will have the form

$$
\exists_{x_{1}, \ldots, x_{7}, y, y_{1}, \ldots, y_{6}} \text { STRAT } \wedge \text { ARITH } \wedge \text { MAX } \wedge \text { LOGSTARCOND . }
$$

We use a number of auxiliary predicates to define $A$, some of which depend on $x_{1}, \ldots, y_{6}$. All variables are distinct unless otherwise stated.

$$
\begin{gathered}
S\left(z_{1}, \ldots, z_{7}\right): \neg(\exists w)\left(w \sim z_{1} \wedge \cdots \wedge w \sim z_{7}\right), \\
N_{1}=\left\{z: x_{1} \sim z \wedge \cdots \wedge x_{7} \sim z\right\} .
\end{gathered}
$$

Membership in $N_{1}$ is expressible in GRA. We use $y, y_{1}, \ldots, y_{6}$ for the (somewhat technical) task of stratifying $N_{1}$.

STRAT: For all $z_{1} \in N_{1}$ there exist unique $z_{2}, \ldots, z_{6} \in N_{1}$ with $S\left(y, z_{1}, \ldots, z_{6}\right)$. Furthermore, for all $z_{1} \in N_{1}$, there is a unique $i, 1 \leq i \leq 6$ for which there exist $z_{2}, \ldots, z_{6}$ with $S\left(y_{i}, z_{1}, \ldots, z_{6}\right)$. (We shall say $z_{1}$ is associated with $i$ in that case.) Furthermore, if $S\left(y, z_{1}, \ldots z_{6}\right)$ then $z_{1}, \ldots, z_{6}$ are associated with distinct $i$.

Set $N$ equal to the set of $z \in N_{1}$ associated with $i=1$. For $z \in N$ set $z^{(1)}=z$ and $z^{(i)}$ equal to that $w$ associated with $i$ so that $S\left(y, z, w, z_{3}, \ldots, z_{6}\right)$ for some $z_{3}, \ldots, z_{6} \in N_{1}$. On $N$ define the ordered 6-ary predicate (here the $z_{i}$ may be equal)

$$
H\left(z_{1}, \ldots, z_{6}\right): S\left(x, z_{1}^{(1)}, z_{2}^{(2)}, \ldots, z_{6}^{(6)}\right) .
$$

ARITH: There is a labelling $1,2, \ldots, s$ of $N$ so that $H\left(z_{1}, \ldots, z_{6}\right)$ if and only if $z_{1}+z_{2}=z_{3}$ and $z_{4} z_{5}=z_{6}$.

Expression of ARITH in GRA is an elementary exercise in logic. We want to say that $N$ is the largest such set over all $x_{1}, \ldots, y_{6}$. If membership in $N, N^{*}$ is expressible then we write

BIGGER $\left(N, N^{*}\right)$ : There exist $v_{3}, \ldots, v_{7}$ so that, writing $S(x, y)$ for $S\left(x, y, v_{3}, \ldots, v_{7}\right)$ the relation $S$ gives an injection from $N-N^{*}$ to $N^{*}-N$ which is not a bijection.

In an interpretation with $N, N^{*}$ finite sets if BIGGER $\left(N, N^{*}\right)$ then $N^{*}$ is indeed bigger (has more elements) than $N$, though the converse may not hold. Now define

MAX: There do not exist $x_{1}^{*}, \ldots, y_{6}^{*}$ satisfying STRAT and ARITH so that, with $N^{*}$ defined analogously to $N$, BIGGER $\left(N, N^{*}\right)$.

Let tower $(x)$ be the number-theoretic function defined inductively by tower $(1)=2$ and tower $(x+1)=2^{\text {tower( }(x)}$ so that tower $(x)$ is an exponential tower of twos of height $x$. On $N$ we develop arithmetic via the Godel $\beta$-function and let $\operatorname{TOWER}(x, y)$ be the predicate with interpretation $y=$ tower $(x)$. Define a unary predicate

$$
\operatorname{PSEUDOLOGSTAR}(x):(\exists y) \operatorname{TOWER}(x, y) \wedge \neg(\exists y) \operatorname{TOWER}(x+1, y) .
$$


Finally, still quantifying over $N$

$$
\begin{aligned}
& \text { LOGSTARCOND: PSEUDOLOGSTAR }(x) \rightarrow(\exists q) \\
& \qquad(x=100 q+1 \vee x=100 q+2 \vee \cdots \vee x=100 q+50)) .
\end{aligned}
$$

This defines the statement $A$.

We now proceed to properties of random graphs. For any $G$ set

$$
N(y)=\{z:\{y, z\} \in G\}, \quad N_{Y}=\bigcap_{y \in Y} N(y)
$$

and let $H^{7}$ be the 7-graph

$$
H^{7}=\left\{Y \in[n]^{7}: N_{Y}=0\right\} .
$$

For every vertex $x$ let $H_{x}$ denote the 6-graph

$$
H_{x}=\left\{Y \in[n]^{6}: Y \cup\{x\} \in H\right\} .
$$

For any $T \subset[n]$ with $x \notin T$ let $H_{x}(T)$ denote the restriction of $H_{x}$ to $T$.

Let us say $G$ has property UNIV (for universal) if for every $T \subset[n]$ with $|T| \leq \log _{8} n$ (recall special notation of $\S 1$ ) and every 6-graph $H$ on $T$ there exists $x \notin T$ so that $H_{x}(T)=H$. Let us say $G$ has property SIZE if for every $t \leq \log _{8} n$ there is a 7-set $X$ with $\left|N_{X}\right|=t$. All probabilities will refer to the random graph $G(n, p)$ with $p$ satisfying $p=n^{-1 / 7} q^{1 / 7}$ and

$$
n^{-1 / \log _{5} n}<q<\log n / \log _{5} n \text {. }
$$

The choice of $\log _{8} n$ above is not "best possible" but simply a function that grows much slower than $\log _{5} n$. (The case $q=1$ already contains the basic argument.)

Claim.

$$
\operatorname{Pr}[U N I V]=1-o(1), \quad \operatorname{Pr}[S I Z E]=1-o(1) .
$$

We outline the arguments which use only standard random graph methods. For $T, H, x$ as above let $A(T, H, x)$ be the event $H_{x}(t)=H$ and let $A(T, H)$ be the event that for no $x, A(T, H, x)$ holds. Fix $T, H$. Our object, toward the first claim, is to bound $\operatorname{Pr}[A(T, H)]$ from above. Call $G$ NICE if

$$
\begin{aligned}
\frac{1}{2} n p^{6}<\left|N_{X}\right| & <2 n p^{6} \text { for all } X \in[n]^{6}, \\
\left|N_{X}\right| & <10 n p^{7} \log n \leq \log ^{3} n \text { for all } X \in[n]^{7} .
\end{aligned}
$$

For fixed $X,\left|N_{X}\right|$ has binomial distribution $B\left(n-6, p^{6}\right)$ and $B\left(n-7, p^{7}\right)$ respectively. The classic Chernoff [3] bounds on the tail of the binomial distribution give that the probability of the above conditions failing goes rapidly to zero, even when multiplied by the $t^{6}$ or $t^{7}$ possible $X$. We use only

$$
\operatorname{Pr}[\text { not } N I C E]=o\left(n^{-\log _{7} n}\right)
$$

and we bound

$$
\operatorname{Pr}[A(T, H)]=o\left(n^{-\log _{7} n}\right)+\operatorname{Pr}[A(t, H) \mid G N I C E] .
$$


We shall show

$$
\operatorname{Pr}[A(T, H)]=o\left(n^{-\log _{7} n}\right) .
$$

It suffices to show $(*)$ conditional on $G N I C E$. We further condition on any particular values for $N(x), x \in T$. Let $R$ denote the union of all $N_{X}$ over all $X \in[T]^{7}$ so that, by NICEness, $R$ has at most $\left(\begin{array}{l}t \\ 7\end{array}\right) \log ^{3} n<\log ^{4} n$ elements. For $X \in[T]^{6}$ let $N_{X}^{\prime}=N_{X}-R$ so that all $N_{X}^{\prime}$ have between $0.4 n p^{6}$ and $2 n p^{6}$ elements.

Let $A^{*}(T, H, x)$ be the event

(i) $N(x) \cap R=\varnothing$.

(ii) If $X \subset T,|X|=6$, and $X \in H$ then $N(x) \cap N_{X}^{\prime}=\varnothing$.

(iii) If $X \subset T,|X|=6$, and $X \notin H$ then $N(x) \cap N_{X}^{\prime} \neq \varnothing$.

Since $|R|<\log ^{4} n, \operatorname{Pr}[N(x) \cap R=\varnothing]>1-p|R|=1-o(1)$. The bounds on $\left|N_{X}^{\prime}\right|$ give

$$
\begin{gathered}
\operatorname{Pr}\left[N(x) \cap N_{X}^{\prime}=\varnothing\right]=(1-p)^{\left|N_{X}^{\prime}\right|}>e^{-3 n p^{7}}=e^{-3 q}, \\
\operatorname{Pr}\left[N(x) \cap N_{X}^{\prime} \neq \varnothing\right]=1-(1-p)^{\left|N_{X}^{\prime}\right|}>1-e^{-0.3 n p^{7}}>0.2 q .
\end{gathered}
$$

Here we see the asymmetry between $q>1$ and $q<1$. Set

$$
\alpha=\min \left(e^{-3 q}, 0.2 q\right) \text {. }
$$

As $R$ and the $N_{X}^{\prime}$ are mutually disjoint the above events are mutually independent and

$$
\operatorname{Pr}\left[A^{*}(T, H, x)\right]>(1-o(1)) \alpha^{\left(\begin{array}{l}
t \\
6
\end{array}\right)}>\alpha^{t^{6}} .
$$

If $A^{*}(T, H, x)$ holds then $A(T, H, x)$ holds so $\operatorname{Pr}[A(T, H, x)]>\alpha^{t^{6}}$. The events $A(T, H, x)$ are mutually independent over all $x \notin T, x \notin N_{X}$ for all $X \subset T,|X|=6$. There are at least $n / 2$ such $x$ so

$$
\operatorname{Pr}[A(T, H)]<(1-\varepsilon)^{n / 2} \text { with } \varepsilon=\alpha^{t^{6}} .
$$

Now $(*)$ follows immediately from the bounds on $q$. There are fewer than $n^{\log _{8} n}$ possible $T, H$. The probability that UNIV does not hold is at most $n^{\log _{8} n} n^{-\log _{7} n}=o(1)$, giving the first claim.

An overview of the above argument may be useful. Essentially $H^{7}$ behaves as a random 6-graph with edge probability $\left(1-p^{7}\right)^{n} \sim e^{-q}$. With $e^{-q}<\frac{1}{2}$, $\operatorname{Pr}\left[H_{x}=H\right] \geq\left(e^{-q}\right)^{\left(\begin{array}{l}t \\ 6\end{array}\right)}$, the minimum achieved when $H=0$. With $e^{-q}>\frac{1}{2}$, $\operatorname{Pr}\left[H_{x}=H\right] \geq\left(1-e^{-q}\right)^{\left(\begin{array}{l}t \\ 6\end{array}\right)}$, the minimum achieved when $H$ is complete. With $q$ near one $\alpha=\min \left[e^{-q}, 1-e^{-q}\right]$ is not too small so $\operatorname{Pr}\left[H_{x}=H\right] \geq \varepsilon=\alpha^{\left(\begin{array}{l}t \\ 6\end{array}\right)}$ is not too small. There are nearly $n$ potential $x$ and the $H_{x}$ are independent so UNIV fails with probability at most $(1-\varepsilon)^{n}$, which is very small. 
SIZE is easier. Fix $n^{-0.1}$ disjoint sets $X_{i}$ of size 7 and let $A_{i}$ be the event that $N_{X_{i}}$ has precisely $t$ elements. Let $B$ be the event that there are no edges on $\bigcup X_{i}$ so

$$
\operatorname{Pr}[B]=(1-p)^{\left(\begin{array}{c}
7 n^{0.1} \\
2
\end{array}\right)}=o(1) .
$$

Conditioning on $B,\left|N_{X_{i}}\right|$ has binomial distribution $B\left(n-7 n^{0.1}, p^{7}\right)$ and so

$$
\begin{aligned}
\operatorname{Pr}\left[A_{i} \mid B\right]= & \left(\begin{array}{c}
n-7 n^{0.1} \\
t
\end{array}\right)\left(p^{7}\right)^{t}\left(1-p^{7}\right)^{n-7 n^{0.1}-t} \\
& \sim\left(q^{t} / t !\right) e^{-q}>n^{-0.01}
\end{aligned}
$$

for $q$ in this range. The $A_{i}$ are then mutually independent so

$$
\operatorname{Pr}\left[\bigwedge \bar{A}_{i} \mid B\right]<\left(1-n^{-0.01}\right)^{n^{0.1}} \ll 1
$$

and

$$
\operatorname{Pr}\left[\bigwedge \bar{A}_{i}\right] \leq \operatorname{Pr}[\bar{B}]+\operatorname{Pr}\left[\bigwedge \bar{A}_{i} \mid B\right] \ll 1 .
$$

Quite easily, $\operatorname{Pr}\left[\Lambda \bar{A}_{i}\right]=o\left(1 / \log _{5} n\right)$. As there are only $\log _{8} n$ values of $T$ to obtain to realize SIZE, $\operatorname{Pr}[\neg S I Z E]=o(1)$.

Finally, we come to the interpretation. Let $G$ be any graph on $n$ vertices satisfying UNIV and SIZE. We claim

(i) If $\log ^{*} n \equiv 25 \bmod 100$ then $G$ satisfies $A$.

(ii) If $\log ^{*} n \equiv 75 \bmod 100$ then $G$ does not satisfy $A$.

As almost all $G$ have properties UNIV and SIZE this will conclude the proof of Theorem 2.

For (i), set $t=6\left[\left(\log _{8} n\right) / 3\right]$. By SIZE there exist $x_{1}, \ldots, x_{6}$ so that $N_{X}$ has size $t$. Label $N_{X}$ by $u^{i}, 1 \leq i \leq 6,1 \leq u \leq t / 6$ arbitrarily. Let $H$ be the 6-graph on $N_{X}$ consisting of the sets $\left\{u^{1}, u^{2}, u^{3}, u^{4}, u^{5}, u^{6}\right\}$. By UNIV there is a vertex $y \notin N_{X}$ so that $H_{y}\left(N_{X}\right)$ is precisely $H^{i}$. Let $H^{+}$be the 6-graph of all $\left\{a^{1}, b^{2}, c^{3}, d^{4}, e^{5}, f^{6}\right\}$ with $a+b=c$ and $d e=f$. Again by UNIV there is an $x$ such that $H_{x}$ is precisely $H^{+}$. These $x_{1}, \ldots, y_{6}$ satisfy STRAT and ARITH. Maybe MAX is not satisfied. However, now take among all choices of $x_{1}, \ldots, y_{6}$ for which STRAT and ARITH are satisfied that one for which $N$ has the biggest size. As some choice gives $|N|=\left[\log _{8} n / 3\right]$ the maximal has $|N|=s \geq\left[\log _{8} n / 3\right]$. This choice will satisfy MAX since $\operatorname{BIGGER}\left(N, N^{*}\right)$ implies that $N^{*}$ is really bigger than $N$. On this $N$, if $\operatorname{TOWER}(x, y)$ then $y=\operatorname{tower}(x)$ so $x \leq \log ^{*} s$. The converse may not hold since TOWER $(x, y)$ requires numbers larger than $y$ in using the Gödel $\beta$-function. However, it is easy to show that no numbers larger than $2^{y}$ are needed. That is: if tower $(x)=y$ and $2^{y}<s$ then TOWER $(x, y)$. Thus PSEUDOLOGSTAR $(x)$ implies $\log ^{*} s-1 \leq x \leq \log ^{*} s$. As $\log _{9} n \equiv 25 \bmod 100, x$ is between 15 and $25 \bmod 100$ and $A$ holds.

For (ii), we know there exist $x_{1}^{*}, \ldots, y_{6}^{*}$ so that STRAT and ARITH are satisfied with $N_{X}^{*}=\left[\log _{8} n / 3\right]$. Suppose $x_{1}, \ldots, y_{6}$ satisfy STRAT and ARITH 
and give a set $N_{X}$ with $\left|N_{X}\right|<\left[\log _{8} n / 3\right]$. Pick $v_{3}, \ldots, v_{6} \in[n]-N_{X}-N_{X}^{*}$ arbitrarily and let $T=N_{X} \cup N_{X}^{*} \cup\left\{v_{3}, \ldots, v_{6}\right\}$ so that $|T| \leq \log _{8} n$. As $N_{X}^{*}$ is bigger than $N_{X}$ there is a function $f$ from $N_{X}-N_{X}^{*}$ into $N_{X}^{*}-N_{X}$ which is an injection but not a surjection. Let $H$ be the 6-graph on $T$ consisting of all sets $\left\{x, f(x), v_{3}, \ldots, v_{6}\right\}, x \in N_{X}-N_{X}^{*}$. By UNIV there is a $v_{7} \notin T$ so that $H=H_{v_{7}}$. Hence $B I G G E R\left(N_{X}, N_{X}^{*}\right)$ is satisfied. (BIGGER is bigger on small sets!) Then MAX is false. That is, any $x_{1}, \ldots, y_{6}$ such that STRAT and ARITH and MAX are satisfied has $N_{X}$ with $s$ elements, $s \geq\left[\log _{8} n / 3\right]$. Hence that element $x$ with PSEUDOLOGSTAR $(x)$ satisfies $\log ^{*} n-10 \leq x \leq \log ^{*} n$. With $\log ^{*} n \equiv 75 \bmod 100, x$ must lie between 65 and $75 \bmod 100$; hence $A$ is false.

The analysis of $\lim f(n, p, A)$ for this $A$ may be extended to $p=n^{-1 / 7-\varepsilon}$, $\varepsilon$ a small constant. With notation as before define properties

$U N I V_{\varepsilon}:$ For every $T \subset[n]$ with $|T| \leq \varepsilon^{-1 / 10}$ and every 6-graph $H$ on $T$ there exists $x \notin T$ so that $H_{x}(T)=H$.

$S I Z E_{\varepsilon}:$ For every $t<\varepsilon^{-1 / 2}$ there is a 7-set $X$ with $\left|N_{X}\right|=t$.

A .a . both $U N I V_{\varepsilon}$ and $S I Z E_{\varepsilon}$ hold. (The exponents of $\varepsilon$ are not best possible.) Furthermore, a.a. $\left|N_{1}\right| \leq \varepsilon^{-1}$ for all $x_{1}, \ldots, x_{7}$. If $x_{1}, \ldots, y_{6}$ are such that $N$ satisfies STRAT and ARITH and MAX then $\varepsilon^{-1 / 11}<|N|<\varepsilon^{-1}$. It follows that:

(i) If $\left[\log ^{*} \varepsilon^{-1}\right] \equiv 25 \bmod 100$ then $\lim f(n, p, A)=1$.

(ii) If $\left[\log ^{*} \varepsilon^{-1}\right] \equiv 75 \bmod 100$ then $\lim f(n, p, A)=0$.

\section{IRRATIONAL EXPONENT}

In this section we prove that $p=n^{-\alpha}, \alpha$ irrational, satisfies the Zero-One Law. Throughout this section $\alpha$ is a fixed irrational, $0<\alpha<1, p=n^{-\alpha}$ and $G$ is the random graph $G(n, p)$. An event occurs almost always-abbreviated a .a.-if its probability approaches unity in $n$. We let $H_{1}, E\left(H_{1}\right)$ denote the vertex and edge sets of $H_{1}$. A rooted graph is a pair $\left(H_{0}, H_{1}\right)$ where $H_{1}$ is a graph and $H_{0}$ is a subset of $H_{1} \cdot\left(H_{0}, H_{1}\right)$ has type $(v, e)$ if $v=\left|H_{1}-H_{0}\right|$ and $e$ is the number of $\{x, y\} \in E\left(H_{1}\right)$ with $y \notin H_{0}$. (Basically, the edges inside $H_{0}$ are to be disregarded.) We employ the interval notation: $\left[H_{0}, H_{1}\right]$ is the set of $H, H_{0} \subset H \subset H_{1} ;\left(H_{0}, H_{1}\right],\left[H_{0}, H_{1}\right),\left(H_{0}, H_{1}\right)$ are the same except $H_{0}, H_{1}$, and both $H_{0}$ and $H_{1}$ respectively are to be excluded from the interval.

Definitions. $\left(H_{0}, H_{1}\right)$ is dense if it has type $(v, e)$ and $e / v>\alpha^{-1}$.

$\left(H_{0}, H_{1}\right)$ is sparse if it has type $(v, e)$ and $e / v<\alpha^{-1}$.

$\left(H_{0}, H_{1}\right)$ is safe if $\left(H_{0}, H\right)$ is sparse for all $H \in\left(H_{0}, H_{1}\right]$.

$\left(H_{0}, H_{1}\right)$ is rigid if $\left(H, H_{1}\right)$ is dense for all $H \in\left[H_{0}, H_{1}\right)$.

$\left(H_{0}, H_{1}\right)$ is hinged if it is safe but for all $H \in\left(H_{0}, H_{1}\right)\left(H, H_{1}\right)$ is not safe.

We now give some elementary consequences of these definitions. When $H \in$ $\left(H_{0}, H_{1}\right)$ and $\left(H_{0}, H\right),\left(H, H_{0}\right),\left(H_{0}, H_{1}\right)$ have types $\left(v^{\prime}, e^{\prime}\right),\left(v^{\prime \prime}, e^{\prime \prime}\right),(v, e)$ 
respectively, counting gives

$(* *) \quad v=v^{\prime}+v^{\prime \prime}, \quad e=e^{\prime}+e^{\prime \prime}$.

(A1) If $\left(H_{0}, H\right)$ and $\left(H, H_{1}\right)$ are sparse then $\left(H_{0}, H_{1}\right)$ is sparse.

(A2) If $\left(H_{0}, H\right)$ and $\left(H, H_{1}\right)$ are dense then $\left(H_{0}, H_{1}\right)$ is dense.

Proofs. Immediate from $(* *)$.

(B1) If $\left(H_{0}, H_{1}\right)$ is sparse then $\left(H, H_{1}\right)$ is safe for some $H \in\left[H_{0}, H_{1}\right)$.

Proof. Take $H \subset H_{1}$ maximal with $\left(H_{0}, H\right)$ dense. (If no such $H$ exists take $\left.H=H_{0}.\right)$ If $\left(H, H_{1}\right)$ is not safe some $\left(H, H^{\prime}\right)$ is dense but (A2) gives $\left(H_{0}, H^{\prime}\right)$ dense, contradicting maximality.

(B2) If $\left(H_{0}, H_{1}\right)$ is dense then $\left(H_{0}, H\right)$ is rigid for some $H \in\left(H_{0}, H_{1}\right]$.

Proof. Take $H \supset H_{0}$ minimal with $\left(H, H_{1}\right)$ sparse. (If no such $H$ exists take $H=H_{1}$.) If $\left(H_{0}, H\right)$ is not rigid some $\left(H^{\prime}, H\right)$ is sparse but (A1) gives $\left(H^{\prime}, H_{1}\right)$ sparse, contradicting maximality.

(C) If $\left(H_{0}, H_{1}\right)$ is hinged of type $(v, e), H \in\left(H_{0}, H_{1}\right)$, and $\left(H_{0}, H\right)$ has type $\left(v^{\prime}, e^{\prime}\right)$ then $e^{\prime} / v^{\prime} \leq e / v$.

Proof. If not, $e^{\prime} / v^{\prime}>e / v$ and $\left(H, H_{1}\right)$ as type $\left(e-e^{\prime}\right) /\left(v-v^{\prime}\right)<e / v$ and so is sparse. (B1) gives $H^{*} \in\left[H, H_{1}\right)$ with $\left(H^{*}, H_{1}\right)$ safe, contradicting definition of hinged.

(D) If $\left(H_{0}, H_{1}\right)$ is dense and $X \cap H_{1}=0$ then $\left(H_{0} \cup X, H_{1} \cup X\right)$ is dense.

Proof. Let $\left(H_{0}, H_{1}\right)$ have type $(v, e)$. Then $\left(H_{0} \cup X, H_{1} \cup X\right)$ has type $\left(v, e^{\prime}\right)$ where, as every edge counted in $\left(H_{0}, H_{1}\right)$ is still counted in $\left(H_{0} \cup X, H_{1} \cup X\right)$, $e \leq e^{\prime}$

(E) If $\left(H_{0}, H\right)$ and $\left(H, H_{1}\right)$ are rigid then $\left(H_{0}, H_{1}\right)$ is rigid.

Proof. Let $H^{*} \in\left[H_{0}, H_{1}\right)$. As $\left(H_{0}, H_{1}\right)$ is rigid $\left(H \cap H^{*}, H\right)$ is dense so $\left(H^{*}, H \cup H^{*}\right)$ is dense, applying (D) with $X=H^{*}-H$. As $\left(H, H_{1}\right)$ is rigid, $\left(H \cup H^{*}, H_{1}\right)$ is dense. By $(\mathrm{A} 2),\left(H^{*}, H\right)$ is dense.

(F) If $\left(H_{0}, H_{1}\right)$ is hinged and $H \in\left(H_{0}, H_{1}\right)$ then $\left(H, H_{1}\right)$ is rigid.

Proof. If not, $\left(H^{\prime}, H_{1}\right)$ is sparse for some $H^{\prime} \in\left[H, H_{1}\right)$. By $(\mathrm{B}),\left(H^{\prime \prime}, H_{1}\right)$ is safe for some $H^{\prime \prime} \in\left[H, H_{1}\right)$, contradicting the definition of hinged.

(G) If $\left(H_{0}, H_{1}\right)$ is rigid and $X \cap H_{1}=\varnothing$ then $\left(H_{0} \cup X, H_{1} \cup X\right)$ is rigid.

Proof. Let $H^{*} \in\left(H_{0} \cup X, H_{1} \cup X\right]$. As $\left(H_{0}, H_{1}\right)$ is rigid, $\left(H^{*}-X, H_{1}\right)$ is dense, so, by (D), $\left(H^{*}, H_{1} \cup X\right)$ is dense. 
(H) Let $\Omega$ be an arbitrary graph, $H_{0}$ a subset of the vertex set of $\Omega$. Let $H_{i} \subset\left(H_{0}, \Omega\right],\left(H_{0}, H_{i}\right)$ rigid, $1 \leq i \leq s$. Then $\left(H_{0}, H_{1} \cup \cdots \cup H_{s}\right)$ is rigid.

Proof. Assume $s=2$. As $\left(H_{0}, H_{2}\right)$ is rigid, $\left(H_{1} \cap H_{2}, H_{2}\right)$ is rigid so, applying (G) with $X=H_{1}-H_{2},\left(H_{1}, H_{1} \cup H_{2}\right)$ is rigid. As $\left(H_{0}, H_{1}\right)$ is rigid, (E) implies $\left(H_{0}, H_{1} \cup H_{2}\right)$ is rigid.

For $s>2$ we use induction.

Given $\left(H_{0}, H_{1}\right)$ we define the $l$-closure $\mathrm{cl}_{l}\left(H_{0} ; H_{1}\right)$ as the union of all sets $H \in\left(H_{0}, H_{1}\right]$ with $\left(H_{0}, H\right)$ rigid and $\left|H-H_{0}\right| \leq l$. When there is no such $H$ we define $\operatorname{cl}_{l}\left(H_{0} ; H_{1}\right)=H_{0}$. We often write $\mathrm{cl}_{l}\left(H_{0}\right)$ when there is an understood universal set $H_{1}$.

(I) $\left(H_{0}, \mathrm{cl}_{l}\left(H_{0}\right)\right)$ is rigid.

Proof. Immediate from $(\mathrm{H})$.

(J) Suppose $H_{0} \subset H_{1}, y \in H_{1}-H_{0}$ and $\left(H_{0} \cup\{y\}, H_{1}\right)$ is rigid. Set $H=\operatorname{cl}_{\left|H_{1}-H_{0}\right|}\left(H_{0} ; H_{1}\right)$. Assume $y \notin H$. Then $\left(H, H_{1}\right)$ is safe.

Proof. If not $\left(H, H^{\prime}\right)$ is dense for some $H^{\prime} \in\left(H, H_{1}\right]$. By (B2) $\left(H, H^{\prime \prime}\right)$ is rigid for some $H^{\prime \prime} \in\left(H, H^{\prime}\right]$. By (I) $\left(H_{0}, H\right)$ is rigid so by $(\mathrm{E})\left(H_{0}, H^{\prime \prime}\right)$ is rigid which, by definition of closure, would force $H^{\prime \prime} \subset H$, a contradiction.

Definition. Let $\left(H_{0}, H_{1}\right)$ be a rooted graph and $f: H_{0} \rightarrow G$ an injection. We say $g: H_{1} \rightarrow G$ is a set-extension if

(i) $x \in H_{0} \Rightarrow g(x)=f(x)$.

We say $g$ is an extension if, in addition,

(ii) $\{x, y\} \in E\left(H_{1}\right), y \notin H_{0} \Rightarrow\{g(x), g(y)\} \in E(G)$. $\subset B$.

We say $g$ is contained in $B$ (equivalently, $B$ contains $g$ ) if $g\left(H_{1}-H_{0}\right)$

Remark. For technical reasons we do not require $f$ to map edges into edges.

Definition. $N\left(f, H_{0}, H_{1}\right)$ is the number of extensions $g$.

Theorem 3. Let $\left(H_{0}, H_{1}\right)$ be safe. There exist $c_{1}, c$ so that a.a. for all $f: H_{0} \rightarrow$ G

$$
n^{\nu-\alpha e}(\log n)^{-c}<N\left(f, H_{0}, H_{1}\right)<c_{1} n^{\nu-\alpha e} .
$$

Proof. If $\left(H_{0}, H_{1}\right)$ is not hinged let $H \in\left(H_{0}, H_{1}\right)$ so that $\left(H, H_{1}\right)$ is safe. As $\left(H_{0}, H_{1}\right)$ is safe, $\left(H_{0}, H_{1}\right)$ is safe a fortiori. Each $f: H_{0} \rightarrow G$ can be extended to $g: H \rightarrow G$ in $N\left(f, H_{0}, H\right)$ ways and thence to $h: H_{1} \rightarrow G$ in $N\left(g, H, H_{1}\right)$ ways so the result follows by induction on $\left|H_{1}-H_{0}\right|$. Henceforth, we assume $\left(H_{0}, H_{1}\right)$ hinged.

We first show the lower bound. A pair $(f, B)$ with $f: H_{0} \rightarrow G$ and $B \subset G$ is called bad if there is no extension $g: H_{1} \rightarrow G$ contained in $B$. 
Lemma 1. There is a constant $k>0$ with the following property. Set $m=k n^{\alpha e / v}$ and let $f: H_{0} \rightarrow G, B \subset G$ with $|B|=m$ be fixed. Then

$$
\operatorname{Pr}[(f, B) \text { bad }]<\frac{1}{2} \text {. }
$$

Proof. For convenience delete $f\left(H_{0}\right)$ from $B$, adjusting $k$ if necessary, so that $f\left(H_{0}\right) \cap B=\varnothing$.

For each $S \subset B$ with size $\left|H_{1}-H_{0}\right|$ specify arbitrarily a set-extension $g$ with $g\left(H_{1}-H_{0}\right)=S$. Let $X_{S}=1$ if $g_{S}$ is an extension; otherwise zero. Let $X=\sum X_{S}$. Then $E\left[X_{S}\right]=p^{e}$ and

$$
E(X)=\left(\begin{array}{c}
m \\
v
\end{array}\right) p^{e}>k^{v} / v !
$$

We want $\operatorname{Pr}[X=0]<\frac{1}{2}$ for which we calculate the second moment.

$$
\begin{aligned}
\operatorname{Var}(X) & =\sum_{S} \operatorname{Var}\left(X_{S}\right)+\sum_{S \neq T} \operatorname{Cov}\left(X_{S}, X_{T}\right) \\
& \leq E(X)+\sum_{j=0}^{\nu-1} \sum_{|S \cap T|=j} \operatorname{Cov}\left(X_{S}, X_{T}\right) .
\end{aligned}
$$

When $j=0 \quad X_{S}, X_{T}$ are independent and $\operatorname{Cov}\left(X_{S}, X_{T}\right)=0$. Otherwise

$$
\operatorname{Cov}\left(X_{S}, X_{T}\right) \leq E\left(X_{S} X_{T}\right)=p^{u}
$$

Here $u$ is the number of pairs which are images of $\{x, y\} \in E\left(H_{1}\right), y \notin H_{0}$ under $g_{S}$ or $g_{T}$. As $g_{S}, g_{T}$ are bijections there are $e$ such pairs in each case so $u=2 e-u^{\prime}$ where $u^{\prime}$ is the number of pairs in both categories. Set $H=$ $g_{S}^{-1}(S \cap T)$ so that $|H|=j$. Then $u^{\prime}$ counts at most edges $\{x, y\} \in E\left[H \cup H_{0}\right]$ with $y \notin H_{0}$. By $(\mathrm{C})$-using here that $\left(H_{0}, H_{1}\right)$ is hinged- $u^{\prime} / j \leq e / v$.

so

$$
\sum_{|S \cap T|=j} \operatorname{Cov}\left(X_{S}, X_{T}\right) \leq m^{2 v-j} p^{2 e-j(e / v)}=\left[m^{v} p^{e}\right]^{2-j / v}
$$

$$
\operatorname{Var}(X) \leq k^{v} / v !+\sum_{j=1}^{v-1}\left(k^{v}\right)^{2-j / v}<\frac{1}{2} E(X)^{2},
$$

when $k$ is chosen sufficiently large. By Chebyshev's Inequality

$$
\operatorname{Pf}[(f, B) b a d] \leq \operatorname{Pr}[X=0] \leq \operatorname{Var}(X) / E(X)^{2} \leq \frac{1}{2}
$$

Lemma 2. Fix $f: H_{0} \rightarrow G, C \subset G$ with $|C|=k^{\prime} n^{\alpha e / v} \log n$. For $k^{\prime}$ sufficiently large a.a.

$$
\operatorname{Pr}[(f, C) b a d]>\frac{1}{2} n^{-\left|H_{0}\right|} \text {. }
$$

Proof. Split $C$ into $k^{\prime \prime} \log n$ disjoint $B_{i}$ of size $m$ given by Lemma 1 . For $(f, C)$ to be bad all $\left(f, B_{i}\right)$ must be bad. But the events " $f\left(, B_{i}\right)$ bad" involving disjoint edge sets are mutually independent, so

$$
\operatorname{Pr}[(f, C) \text { bad }]<2^{-k^{\prime \prime} \log n},
$$


which can be made smaller than any power of $n$.

With $k^{\prime}$ as above there are only $n^{\left|H_{0}\right|}$ injections $f: H_{0} \rightarrow G$ so a.a. for every $f$ at most half the $(f, C)$ are bad. Set $M=|C|=k^{\prime} n^{\alpha e / v} \ln n$ for convenience. Each extension $g: H_{1} \rightarrow G$ is contained in $\left(\begin{array}{c}n-v \\ M-v\end{array}\right) \sim\left(\begin{array}{c}n \\ m\end{array}\right)(M / n)^{v}$ $m$-sets. Together at most $\left|N\left(f, H_{0}, H_{1}\right)\right|\left(\begin{array}{c}n-v \\ M-v\end{array}\right) \quad M$-sets contain an extension but at least $\frac{1}{2}\left(\begin{array}{l}n \\ M\end{array}\right)$ do. Thus

$$
\begin{gathered}
\left|N\left(f, H_{0}, H_{1}\right)\right|\left(\frac{M}{n}\right)^{v} \geq \frac{1}{2}, \\
\left|N\left(f, H_{0}, H_{1}\right)\right| \geq \frac{1}{2}\left(\frac{n}{M}\right)^{v}>n^{v-\alpha e}(\ln n)^{-v+o(1)},
\end{gathered}
$$

completing the proof of the lower bound of Theorem 3 .

Conjecture. A.a. for all $f N\left(f, H_{0}, H_{1}\right)>c^{\prime} n^{\nu-\alpha e}$ where $c^{\prime}$ depends only on $H_{0}$ and $H_{1}$.

The upper bound to Theorem 3 also requires some preliminary lemmas. Two extensions $g_{1}, g_{2}$ of $f$ on $\left(H_{0}, H_{1}\right)$ are called disjoint if

$$
g_{1}\left(H_{1}-H_{0}\right) \cap g_{2}\left(H_{1}-H_{0}\right)=\varnothing .
$$

Lemma 3. Let $\left(H_{0}, H_{1}\right)$ be dense. There exist $K$ so that a.a. for all $f: H_{0} \rightarrow G$ there do not exist $K$ disjoint extensions $g_{1}, \ldots, g_{K}$.

Proof. Let $c=\left|H_{0}\right|$ and $\left(H_{0}, H_{1}\right)$ have type $(v, e)$. Pick $K$ so that $c+$ $K(v-\alpha e)<0$. There are less than $n^{c}\left(n^{v}\right)^{K}\left(f, g_{1}, \ldots, g_{K}\right)$ with $g_{i}$ disjoint set-extensions of $f$. Each $g_{i}$ is an extension with probability $p^{e}$ and since they are disjoint they are all extensions with probability $p^{e K}$ so the probability of $\left(f, g_{1}, \ldots, g_{K}\right)$ existing with all $g_{i}$ extensions is less than $n^{c+v K-\alpha e K}=o(1)$.

Lemma 4. Let $\left(H_{0}, H_{1}\right)$ be rigid. There exist $K$ so that a.a. for all $f: H_{0} \rightarrow G$ there do not exist $K$ extensions $g_{1}, \ldots, g_{K}$.

Proof. For all $H \in\left(H_{0}, H_{1}\right),\left(H, H_{1}\right)$ is dense so we may pick $K_{0}$ so that Lemma 3 holds for all $\left(H, H_{1}\right)$. By Erdós and Rado [4] we may pick $K$ so that given any $K$ functions on $v=\left|H_{1}-H_{0}\right|$ points there is a $\Delta$-system of $K_{0}$ functions. These $K_{0}$ functions would be disjoint extensions of the common root $H$.

Now we fix $f: H_{0} \rightarrow G$ and bound $s^{\prime}=N\left(f, H_{0}, H_{1}\right)$. from above. For any $h \in H_{1}, y \in G \quad\left(H_{0} \cup\{h\}, H_{1}\right)$ is rigid (F) so by Lemma 4 there are at most $K$ extensions $g$ with $g(h)=y$. Each extension $g$ is disjoint from all but at most $K\left|H_{0}\right|^{2}$ extensions and so there is a set of $s=s^{\prime} /\left(K\left|H_{0}\right|^{2}+1\right)$ disjoint extensions.

There are less than $n^{c}\left(\begin{array}{l}n \\ v\end{array}\right)^{s} / s$ ! pairs consisting of an $f: H_{0} \rightarrow G$ and a set $\left\{g_{1}, \ldots, g_{s}\right\}$ of disjoint set-extensions of $f$. The probability that all $g_{i}$ are extensions is, as before, $p^{e s}$. Thus the probability of $f,\left\{g_{1}, \ldots, g_{s}\right\}$ existing is at most

$$
n^{c} n^{v s} p^{e s} / s !<n^{c}\left(2.72 n^{v} p^{e / s}\right)^{s} \ll 1
$$


when $s=3 n^{v-\alpha e}$. Thus, a.a.,

$$
N\left(f, H_{0}, H_{1}\right) \leq 3\left(K\left|H_{0}\right|^{2}+1\right) n^{v-\alpha e},
$$

completing the proof of Theorem 3 .

Lemma 5. For all $r, l$ there is a $K$ so that a.a. $\left|\operatorname{cl}_{l}\left(G_{0}\right)\right| \leq K$ for all $G_{0} \subset G$, $\left|G_{0}\right| \leq r$.

Proof. There are a bounded (Lemma 4) number of extensions for each rigid $\left(H_{0}, H_{1}\right)$ with $\left|H_{0}\right|=\left|G_{0}\right|$ and only a finite number of such $\left(H_{0}, H_{1}\right)$ up to isomorphism.

Lemma 6. For all $r, l_{1}, l_{2}$ there is an $l$ so that a.a.

$$
\mathrm{cl}_{l_{1}}\left(\mathrm{cl}_{l_{2}}\left(G_{0}\right)\right) \subseteq \mathrm{cl}_{l}\left(G_{0}\right)
$$

for all $G_{0} \subset G,\left|G_{0}\right| \leq r$.

Proof. Set $l=K+l_{1}$ where $\left|\mathrm{cl}_{l_{2}}\left(G_{0}\right)\right| \leq K$ by Lemma 5. If $y \in \mathrm{cl}_{l_{1}}\left(\operatorname{cl}_{l_{2}}\left(G_{0}\right)\right)$ then $y \in G^{\prime}, \quad\left|G^{\prime}-\mathrm{cl}_{l_{2}}\left(G_{0}\right)\right| \leq l_{1},\left(\mathrm{cl}_{l_{2}}\left(G_{0}\right), G^{\prime}\right)$ rigid. From (I) and $(\mathrm{E})$, $\left(G_{0}, G^{\prime}\right)$ is rigid, $\left|G^{\prime}-G_{0}\right| \leq l$ so $y \in \mathrm{cl}_{l}\left(G_{0}\right)$.

Let $x_{1}, \ldots, x_{r} \in G$. By $\operatorname{cl}_{l}\left(x_{1}, \ldots, x_{r}\right)$ we shall mean the graph on $\operatorname{cl}_{l}\left(\left\{x_{1}, \ldots, x_{r}\right\}\right)$ with points $x_{1}, \ldots, x_{r}$ specified. We say $\left(x_{1}, \ldots x_{r}\right)$, $\left(x_{1}^{\prime}, \ldots, x_{r}^{\prime}\right)$ have the same $l$-type if $\mathrm{cl}_{l}\left(x_{1}, \ldots, x_{r}\right) \cong \mathrm{cl}_{l}\left(x_{1}^{\prime}, \ldots, x_{r}^{\prime}\right)$, the isomorphism sending $x_{i}$ to $x_{i}^{\prime}$. By Lemma 5 the family $\widehat{L}_{r, l}$ of possible $l$-types is a .a. bounded. Each one can be considered as a graph $H_{1}$ with specified vertices $h_{1}, \ldots, h_{r}$ such that $H_{1}=\operatorname{cl}_{l}\left(\left\{h_{1}, \ldots, h_{r}\right\} ; H_{1}\right)$.

Now we are ready to deal with first order sentences. Let $\phi\left(x_{1}, \ldots, x_{r}\right)$, as customary, denote a formula in GRA with free variables $x_{1}, \ldots, x_{r}$.

Theorem 4. For every $\phi\left(x_{1}, \ldots, x_{r}\right)$ there is an $l$ so that a.a. for all $x_{1}, \ldots, x_{r}$ $\in G \phi\left(x_{1}, \ldots, x_{r}\right)$ depends only on $\mathrm{cl}_{l}\left(x_{1}, \ldots, x_{r}\right)$. That is, there exist $l$, $\mathscr{A} \subseteq \widehat{L}_{r, l}$ so that a.a.

$$
(* * *) \quad G \vDash \phi\left(x_{1}, \ldots, x_{r}\right) \Leftrightarrow \operatorname{cl}_{l}\left(x_{1}, \ldots, x_{r}\right) \in \mathscr{A} .
$$

Example. Let $\frac{1}{3}+\frac{1}{9} k<\alpha<\frac{1}{3}+\frac{1}{9}(k-1)$ and set

$$
\begin{aligned}
\phi(x, y, z):(\exists w)(\forall a)[a & \sim x \wedge a \sim y \wedge a \sim z \rightarrow(\exists ! b \neq a)(\exists v) \\
b & \sim x \wedge b \sim y \wedge b \sim z \wedge v \sim a \wedge v \sim b \wedge v \sim w],
\end{aligned}
$$

$\phi(x, y, z)$ depends on $\mathrm{cl}_{3 k+1}(x, y, z) . \mathrm{cl}_{l}(x, y, z)$ contains all points $a_{1}, \ldots$, $a_{2 s}$ adjacent to $x, y, z$. (If there are an odd number of such points $G \vDash \neg \phi$.) If $s<k$ then $G \vDash \phi$ since the extension by $w, v_{1}, \ldots, v_{s}$ with $w \sim v_{i} \sim a_{2 i-1}, a_{2 i}$ is safe. When $s \geq k, G \vDash \phi$ if and only if $\operatorname{cl}_{3 k+1}(x, y, z)$ contains points $w, v_{1}, \ldots, v_{s}$ so that, after renumbering, $w \sim v_{i} \sim a_{2 i-1}, a_{2 i}$. An examination 
of $\mathrm{cl}_{3 k}(x, y, z)$ would not suffice since if $\mathrm{cl}_{3 k}(x, y, z)$ consists of $a_{1}, \ldots, a_{2 k}$ joined to $x, y, z$ the desired $w, v_{1}, \ldots, v_{k}$ might exist outside $\operatorname{cl}_{3 k}(x, y, z)$.

Proof. We use induction on the length of $\phi$. Let us say $l, \mathscr{A}$ give $\phi$ if $(* * *)$ holds. Let $\neg, \vee,(\exists y)$ be the only logical connectives. For atomic formulae $x_{i} \sim x_{j}$ or $x_{i}=x_{j}$ simply take $l=0$. If $\phi=\neg \psi$ and $l, \mathscr{A}$ give $\psi$ then $l, \widehat{L}-\mathscr{A}$ give $\phi$. If $l<l^{\prime}$ then $\mathrm{cl}_{l^{\prime}}\left(x_{1}, \ldots, x_{r}\right)$ determines $\mathrm{cl}_{l}\left(x_{1}, \ldots, x_{r}\right)$ so that if $l, \mathscr{A}$ give $\phi$ and $l<l^{\prime}$ there is an $\mathscr{A}^{\prime}$ so that $l^{\prime}, \mathscr{A}^{\prime}$ give $\phi$. If $\phi=\phi_{1} \vee \phi_{2}$ and, by induction, $l_{1}, \mathscr{A}_{1}$ give $\phi_{1}$ and $l_{2}, \mathscr{A}_{2}$ give $\phi_{2}$ then set $l=\max \left(l_{1}, l_{2}\right)$ so that $l, \mathscr{A}_{1}^{\prime}$ give $\phi_{1}$ and $l, \mathscr{A}_{2}^{\prime}$ give $\phi_{2} \cdot \phi$ is then given by $l, \mathscr{A}_{1}^{\prime} \cup \mathscr{A}_{2}^{\prime}$. This leaves the critical case $\phi\left(x_{1}, \ldots, x_{r}\right):(\exists y) \psi\left(x_{1}, \ldots, x_{r}, y\right)$. By the inductive hypothesis there exist $l, \mathscr{A} \subseteq \widehat{L}_{r+1, l}$ giving $\psi$. Thus

$$
G \vDash \phi\left(x_{1}, \ldots, x_{r}\right) \Leftrightarrow \text { for some } y \in G \operatorname{type}_{l}\left(x_{1}, \ldots, x_{r}, y\right) \in \mathscr{A} \text {. }
$$

It suffices to show the following.

Theorem 5. Let $H_{0}=\left(h_{1}, \ldots, h_{r}, h^{*}\right)$ and let $\left(H_{0}, H_{1}\right) \in \widehat{L}_{r+1, l}$. Then there exists $l^{\prime}$ and $\mathscr{B} \subseteq \widehat{L}_{l^{\prime}, r}$ so that a.a. for all $x_{1}, \ldots, x_{r} \in G$

$$
(\exists y) \operatorname{type}_{l}\left(x_{1}, \ldots, x_{r}, y\right)=\left(H_{0}, H_{1}\right) \Leftrightarrow \operatorname{type}_{l^{\prime}}\left(x_{1}, \ldots, x_{r}\right) \in \mathscr{B} .
$$

Assume Theorem 5 and let $\mathscr{A}$, above, consist of types $T^{1}, \ldots, T^{s}$. For each $T^{i}$ there are $l^{i}, \mathscr{B}^{i}$ satisfying Theorem 5 . We replace all $l^{i}$ by $l=$ $\max \left(l^{1}, \ldots, l^{s}\right)$ and $\mathscr{B}^{i}$ by $\mathscr{B}^{i *}$. Then $\phi$ is given by $l, \mathscr{A}$ where $\mathscr{A}=\mathscr{B}^{1 *} \cup$ $\cdots \cup \mathscr{B}^{s *}$.

To prove Theorem 5 we set

$$
H=\mathrm{cl}_{\left|H_{1}\right|}\left[\left\{h_{1}, \ldots, h_{r}\right\} ; H_{1}\right] \text {. }
$$

Case 1. $h^{*} \in H$. As $\left(\left\{h_{1}, \ldots, h_{r}, h^{*}\right\}, H_{1}\right)$ is rigid by $(\mathrm{E})\left(\left\{h_{1}, \ldots, h_{r}\right\}, H_{1}\right)$ is also rigid and so $H=H_{1}$. Let $l^{\prime}$ be such that $\operatorname{cl}_{l}\left(\mathrm{cl}_{l}(X)\right) \subset \mathrm{cl}_{l^{\prime}}(X)$ for all $X \subset G,|X|=r$. Let $\mathscr{B}$ be the set of all $l^{\prime}$-types $\left(\left(h_{1}, \ldots, h_{r}\right), H_{2}\right)$ so that for some $h^{*} \in H_{2}$

$$
\left(\left(h_{1}, \ldots, h_{r}, h^{*}, \operatorname{cl}_{l}\left(\left\{h_{1}, \ldots, h_{r}, h^{*}\right\} ; H_{2}\right)\right) \cong\left(H_{0}, H_{1}\right) .\right.
$$

If type ${ }_{l}\left(x_{1}, \ldots, x_{r}\right) \in \mathscr{B}$ then with $y$ the image of $h^{*}$ type $_{l}\left(x_{1}, \ldots, x_{r}, y\right)=$ $\left(H_{0}, H_{1}\right)$. Conversely if there exists $y$ with type ${ }_{l}\left(x_{1}, \ldots, x_{r}, y\right)=\left(H_{0}, H_{1}\right)$ then $y \in \operatorname{cl}_{l}\left(x_{1}, \ldots, x_{r}\right)$ so $\mathrm{cl}_{l}\left(x_{1}, \ldots, x_{r}, y\right) \subset \mathrm{cl}_{l^{\prime}}\left(x_{1}, \ldots, x_{r}\right)$ and so $\mathrm{cl}_{l}\left(x_{1}, \ldots, x_{r}\right) \in \mathscr{B}$.

Case 2. $h^{*} \notin H$. We claim

$$
\begin{aligned}
(\exists y) \operatorname{type}_{l}\left(x_{1}, \ldots, x_{r}, y\right)=\left(\left(h_{1}, \ldots, h_{r}, h^{*}\right), H_{1}\right) & \\
& \Leftrightarrow \operatorname{type}_{l}\left(x_{1}, \ldots, x_{r}\right)=\left(\left(h_{1}, \ldots, h_{r}\right) ; H\right) .
\end{aligned}
$$


As type $e_{l}\left(x_{1}, \ldots, x_{r}, y\right)$ determines type $e_{l}\left(x_{1}, \ldots, x_{r}\right), \Rightarrow$ is immediate. Now assume type $e_{l}\left(x_{1}, \ldots, x_{r}\right)=\left(\left(h_{1}, \ldots, h_{r}\right), H\right)$; we must find a "witness" $y$. By $(\mathrm{J})$ the rooted graph $\left(H, H_{1}\right)$ is safe. Fix $f: H \rightarrow G$ with $f\left(h_{i}\right)=x_{i}$ and $\left\{h^{\prime}, h^{\prime \prime}\right\} \in E(H) \Leftrightarrow\left\{f\left(h^{\prime}\right), f\left(h^{\prime \prime}\right)\right\} \in E(G)$. ( $f$ exists by definition of type.) Let $\left(H, H_{1}\right)$ have type $(v, e)$. Theorem 3 bounds the number of extensions $g: H_{1} \rightarrow G$. If $g: H_{1} \rightarrow G$ is an extension, setting $y=g\left(h^{*}\right)$ might not work as $\mathrm{cl}_{l}\left(x_{1}, \ldots x_{r}, y\right)$ might have more vertices or edges than $\left(H_{1}, E\left(H_{1}\right)\right)$. This is critical-we need to find a witness $y$ with the desired special properties and no others.

Let $\mathscr{l}$ be the family of types $\left(\left(h_{1}, \ldots, h_{r}, h^{*}\right), H^{*}\right) \in \widehat{L}_{r+1, l}$ such that $H_{1}$ is a subgraph of $H^{*}$. Let $M\left(H^{*}\right)$ be the number of $y$ so that there is an extension $g: H^{*} \rightarrow G$ of $f$ relative to $\left(H_{0}, H^{*}\right)$ with $g\left(h^{*}\right)=y$. For each $y$ Lemma 4 gives there are at most $K$ such $f$. Let $\left(v^{*}, e^{*}\right)$ denote the type of $\left(H_{0}, H^{*}\right)$. Theorem 3 gives

$$
n^{v^{*}-\alpha e^{*}}(\ln n)^{-c}<M\left(H^{*}\right)<c_{1} n^{v^{*}-\alpha e^{*}}
$$

for adjusted constants $c, c_{1}$. There are $M\left(H_{1}\right)$ y such that $\mathrm{cl}_{l}\left(x_{1}, \ldots, x_{r}, y\right)$ contains $\left(H_{1}, E\left(H_{1}\right)\right)$ as a subgraph. Let $\mathscr{H}^{*}$ be $\mathscr{H}$ with $\left(H_{1}, E\left(H_{1}\right)\right)$ deleted. For each $\left(H^{*}, E\left(H^{*}\right)\right) \in \mathscr{H}^{*}$ there are at most $M\left(H^{*}\right) \quad y$ so that $\mathrm{cl}_{l}\left(x_{1}, \ldots, x_{r}, y\right) \cong\left(H^{*}, E\left(H^{*}\right)\right)$. Thus there are at least

$$
M(H)-\sum_{H^{*} \in \mathscr{H}^{*}} M\left(H^{*}\right)
$$

$y$ with type ${ }_{l}\left(x_{1}, \ldots, x_{r}, y\right)=\left(\left(h_{1}, \ldots, h_{r}\right), H_{1}\right)$.

Let $\left(H^{*}, E\left(H^{*}\right)\right) \in \mathscr{H}^{*}$ have type $\left(v^{*}, e^{*}\right)$. Suppose $H_{1} \varsubsetneqq H^{*}$. Since $\left(\left\{h_{1}, \ldots, h_{r}, h^{*}\right\}, H^{*}\right)$ is rigid so is $\left(H_{1}, H^{*}\right)$, hence $\left(e^{*}-e\right) /\left(v-v^{*}\right)>\alpha^{-1}$ and $v^{*}-e^{*} \alpha<v-\alpha e$. When $H_{1}=H^{*}$ we have $v=v^{*}$ so $e^{*}>e$ and again $v^{*}-e^{*} \alpha<v-\alpha e$. In all cases $M\left(H^{*}\right)$ is much smaller than $M(H)$. Then

$$
\begin{aligned}
M(H)-\sum_{H^{*} \in \mathscr{R}^{*}} M\left(H^{*}\right) & >n^{\nu-\alpha e}(\ln n)^{-c}-\sum c_{1} n^{\nu^{*}-\alpha e^{*}} \\
& =n^{\nu-\alpha e+o(1)}>0 .
\end{aligned}
$$

The witness $y$ exists, giving Case 2, Theorem 5 and Theorem 4 .

Theorem 6. If $\alpha$ is irrational then $p=n^{-\alpha}$ satisfies the Zero-One Law.

Proof. Let $\phi$ be a closed formula. By Theorem 4 there exist $l, \mathscr{A}$ so that a.a .

$$
G \vDash \phi \Leftrightarrow \mathrm{cl}_{l}(\varnothing) \in \mathscr{A} .
$$

A dense rooted graph $\left(\varnothing, H_{1}\right)$ of type $(v, e)$ appears an expected number $n^{v} p^{e}=o(1)$ of times in $G$. For a given $l$ there are only a fixed number of such $H_{1}$ so a.a. no such $H_{1}$ appears. That is, a.a. $\mathrm{cl}_{l}(\varnothing)=\varnothing$. Hence a.a.

$$
G \vDash \phi \Leftrightarrow \varnothing \in \mathscr{A} \text {. }
$$


Either $\varnothing \in \mathscr{A}$ or $\varnothing \notin \mathscr{A}$ so either a.a. $G \vDash \phi$ or a.a. $G \vDash \neg \phi$.

\section{THRESHOLD SPECTRA}

The analysis of $\S 4$ was essentially static. Now consider $A$ fixed and consider the property $A$ over the "evolution" of the random graph. We would like to describe this evolution in terms of a spectrum of threshold functions, though the results of $\S 3$ force us to use some care.

Call $a>0$ a point of continuity if there is an $\varepsilon>0$ and $\delta \in\{0,1\}$ so that if $n^{-a-\varepsilon}<p<n^{-a+\varepsilon}$ then $\operatorname{Lim} f(n, p, A)=\delta$. Otherwise, call $a$ an evolutionary discontinuity and define the threshold spectrum $\operatorname{Spec}(A)$ as the set of such $a$. From the methods of $\S 4$ it follows that all irrational $a$ are points of continuity and that $\operatorname{Spec}(A)$ is a closed nowhere dense set of rational numbers. Many interesting $A$ analyzed by Erdős, Rényi, and others have threshold spectrum consisting of a single point. For monotone $A$, not necessarily in GRA, the existence of a threshold function is shown by Bollobás and Thomason [2] to follow from purely combinatorial argument. The analysis concluding $\S 3$ shows that $\operatorname{Spec}(A)$ may have a limit point.

A set of real numbers $S$ is called scattered if it is closed and the sequence $S=S_{0}, S_{1}, \ldots$, with $S_{i+1}$ the set of limit points of $S_{i}$, has $S_{m}=\varnothing$ for some $m$.

Theorem 7. $\operatorname{Spec}(A)$ is scattered.

We outline the argument, which requires proving Theorem 6 in a manner uniform on $\alpha$. The example following the statement of Theorem 4 illustrates that the $l$ of Theorem 4 need not be bounded uniformly in $\alpha$. The essential induction of Theorem 4 becomes the following.

Lemma 7. For every $\phi\left(x_{1}, \ldots, x_{r}\right)$ there is a scattered $S=\operatorname{Scat}(\phi) \subset Q$ so that for all $a, b$ with $[a, b] \cap S=\varnothing$ there is an $l, \mathscr{A}$ so that if $n^{-b}<p<n^{-a}$ then a.a. for all $x_{1}, \ldots, x_{r} \in G$

$$
G \vDash \phi\left(x_{1}, \ldots, x_{r}\right) \Leftrightarrow \operatorname{cl}_{l}\left(x_{1}, \ldots, x_{r}\right) \in \mathscr{A} .
$$

The proof is by induction. We let $S=\{0,2\}$ for the null statement to set up boundaries. The cases $\phi=\neg \psi$ and $\phi=\phi_{1} \vee \phi_{2}$ are simple; again the critical case is

$$
\phi(x):(\exists y) \psi(x, y) \text {, }
$$

where we abbreviate $x_{1}, \ldots, x_{r}$ as $x$. Let $S_{0}=\operatorname{Scat}(\psi)$. We expand $S_{0}$ in four stages.

For each consecutive $a, a^{\prime} \in S_{0}$ add a doubly infinite monotonic sequence $b_{n}, n \in Z$, of rationals from $a$ to $a^{\prime}$. To be explicit: $b_{n}=a^{\prime}-\left(a^{\prime}-a\right) 2^{-n-1}$ for $n \geq 0 ; b_{n}=a+\left(a^{\prime}-a\right) 2^{n-1}$ for $n<0$. Let $S_{1}$ be the enlarged set. For every consecutive $b, b^{\prime} \in S_{1}$ (note: the $a \in S_{0}$ are now limit points so $b=b_{n}$, 
$\left.b^{\prime}=b_{n+1}\right)$ find, by induction, $l, \mathscr{A}$ so that for $n^{-b^{\prime}}<p<n^{-b}$ a.a. for all $x$

$(* * * *) \quad G \vDash \phi(x) \Leftrightarrow(\exists y) \mathrm{cl}_{l}(x, y) \in \mathscr{A}$.

When $\left(H_{0}, H_{1}\right) \in \mathscr{A}, H_{0}=\left(h_{1}, \ldots, h_{r}, h^{*}\right)$ we consider

$$
H=\mathrm{cl}_{\left|H_{1}\right|}\left[\left\{h_{1}, \ldots, h_{r}\right\} ; H_{1}\right]
$$

as a function of $\alpha \in\left[b^{\prime}, b\right]$. Fix $M$ with $M \geq r+l$ and $M \geq\left|H_{1}\right|$ for all $\left(H_{0}, H_{1}\right) \in \mathscr{A}$. In $\left[b^{\prime}, b\right]$ add all rationals $x / y$ with $y \leq\left(\begin{array}{c}M \\ 2\end{array}\right)$, giving $S_{2}$. The value of $H$ can change only at $\alpha \in S_{2}$. For consecutive $c, c^{\prime} \in S_{2} \quad(* * * *)$ still holds and $H$ is well defined over $\alpha \in\left(c, c^{\prime}\right)$. When $h^{*} \notin H$

$$
(\exists y) \mathrm{cl}_{l}(x, y)=\left(H_{0}, H_{1}\right) \Leftrightarrow \mathrm{cl}_{l}(x)=\left(\left(h_{1}, \ldots, h_{r}\right) ; H\right) .
$$

For each consecutive $c, c^{\prime} \in S_{2}$ add a doubly infinite sequence $d_{n}$ of rationals from $c$ to $c^{\prime}$ giving $S_{3}$. Let $d, d^{\prime}$ be consecutive elements of $S_{3}$. All rooted graphs $\left(H, H_{1}\right)$ with $\left|H_{1}\right| \leq r+l$ with type $(v, e)$ that are dense over $\alpha \in\left(c, c^{\prime}\right)$ will have $v-\alpha e<-\varepsilon$ where $\varepsilon$ is constant over all $\alpha \in\left[d, d^{\prime}\right]$. Lemma 3 gives a $K$ so that no $f: H \rightarrow G$ has more than $K$ disjoint extensions to $g: H_{1} \rightarrow G$. Lemmas 4, 5, and 6 follow and there is an $l^{*}$ so that if $n^{-d^{\prime}}<p<n^{-d}$ then a.a. $\operatorname{cl}_{l}\left(\operatorname{cl}_{l}(x)\right) \subset \operatorname{cl}_{l^{*}}(X)$ for all $X \subset G,|X|=r$. When $h^{*} \in H$ the statement $(\exists y) \mathrm{cl}_{l}(x, y)=\left(H_{0}, H_{1}\right)$ is determined by $\mathrm{cl}_{l^{*}}(x)$. For every consecutive $d, d^{\prime}$ add all rational $x / y \in\left(d, d^{\prime}\right)$ with $y \leq\left(\begin{array}{c}r+l^{*} \\ 2\end{array}\right)$, giving $S_{4}$.

Assume $[A, B] \cap S_{4}=\varnothing$. There is an $\varepsilon>0$ so that $|\alpha-x / y|>\varepsilon$ for all $x, y$ above. As before, there is a constant $K$ so that if $n^{-B}<p<n^{-A}$ then a.a. $\left|\operatorname{cl}_{l^{*}}(X)\right| \leq K$ for all $X \subset G,|X|=r$. Then

$$
(\exists y) \mathrm{cl}_{l}(x, y)=\left(H_{0}, H_{1}\right) \Leftrightarrow \mathrm{cl}_{l^{*}}(x) \in \mathscr{B}
$$

where $\mathscr{B}$ is that set of possible closures that have an $h^{*}$ generating $H_{1}$.

As $\mathscr{A}$ is a finite set and we can do this for all $\left(H_{0}, H_{1}\right) \in \mathscr{A}$, there is an $l^{*}, \mathscr{B}^{*}$ so that with $n^{-B}<p<n^{-A}$ a.a.

$$
(\exists y) \mathrm{cl}_{l}(x, y) \in \mathscr{A} \Leftrightarrow \mathrm{cl}_{l *}(x) \in \mathscr{B}
$$

and so we may set $\operatorname{Scat}(\phi)=S_{4}$.

$S$ is precisely the set of accumulation points of $S_{4}$ so $S_{4}$ is scattered by induction, completing Lemma 7.

Now let $A$ be a closed sentence of GRA and let $S=\operatorname{Scat}(A)$ be given by Lemma 7. Suppose $\alpha \notin S$. As $S$ is closed some $[\alpha-\varepsilon, \alpha+\varepsilon] \cap S=\varnothing$. Hence there are $l, \mathscr{A}$ so that for all $p$ with $n^{-\alpha-\varepsilon}<p<n^{-\alpha+\varepsilon}$ a.a.

$$
G \vDash A \Leftrightarrow \mathrm{cl}_{l}(\varnothing) \in \mathscr{A} \text {. }
$$

But, as before, a.a. $\mathrm{cl}_{l}(\varnothing)=\varnothing$. If $\varnothing \in \mathscr{A}$ then a.a. $G \vDash A$, otherwise a .a. $G \vDash \neg A$. Hence $\alpha \notin \operatorname{Spec}(A)$. $\operatorname{Spec}(A)$ is a closed subset of a scattered set, hence it is scattered. 
A full characterization of the threshold spectra of first order statements has proven elusive. In particular, we do not know if $\operatorname{Spec}(A)$ may contain an infinite number of limit points.

\section{BIBLIOGRAPHY}

1. B. Bollobás, Random graphs, Academic Press, New York, 1985.

2. B. Bollobás and A. G. Thomason, Threshold functions, Combinatorica 7 (1986), 35-38.

3. H. Chernoff, A measure of asymptotic efficiency for tests of a hypothesis based on a sum of observations, Ann. Math. Stat. 23 (1952), 493-509.

4. P. Erdos and R. Rado, Intersection theorems for systems of sets, J. London Math. Soc. 35 (1960), 85-90.

5. P. Erd6s and A. Rényi, On the evolution of random graphs, Publ. Math. Inst. Hungar. Acad. Sci. 5 (1960), 17-61.

6. R. Fagin, Probabilities on finite models, J. Symbolic Logic 41 (1976), 50-58.

7. Y. V. Glebskii, D. I. Kogan, M. I. Liogonkii and V. A. Talanov, Range and degree of realizability of formulas in the restricted predicate calculus, Cybernetics 5, 142-154.

8. I. Marcus, Minimal models of one function symbol, Israel J. Math. 18 (1974), 117-130.

9. M. Kaufman and S. Shelah, On random models of finite powers and monadic logic, Discrete Math. 54 (1985), 285-293.

Department of Mathematics, Hebrew University, Jerusalem, IsRael

Department of Mathematics, SUNy at Stony Brook, Stony Brook, New York 11794

Courant Institute, 251 Mercer, New York, New York 10012 (Current address of Joel Spencer) 